\title{
Uncertainty in marine weather routing
}

\author{
Thomas Dickson ${ }^{\mathrm{a}}$, Helen Farr ${ }^{\mathrm{b}}$, David Sear ${ }^{\mathrm{c}}$, James I R Blake \\ ${ }^{a}$ Fluid Structure Interactions, Faculty of Engineering and Physical Sciences, Boldrewood Innovation \\ Campus, University of Southampton, Southampton SO16 7QF, United Kingdom \\ ${ }^{b}$ Maritime Archaeology, Faculty of Arts and Humanities, University of Southampton Avenue Campus, \\ Highfield, Southampton, SO17 1BF, United Kingdom \\ ${ }^{c}$ Physical Geography, Faculty of Environmental and Life Sciences, Highfield Campus, University of \\ Southampton, Southampton S017 1BJ, United Kingdom
}

\begin{abstract}
Weather routing methods are essential for planning routes for commercial shipping and recreational craft. This paper provides a methodology for quantifying the significance of numerical error and performance model uncertainty on the predictions returned from a weather routing algorithm. The numerical error of the routing algorithm is estimated by solving the optimum path over different discretizations of the environment. The uncertainty associated with the performance model is linearly varied in order to quantify its significance. The methodology is applied to a sailing craft routing problem: the prediction of the voyaging time for an ethnographic voyaging canoe across long distance voyages in Polynesia. We find that the average numerical error is an order of magnitude smaller than the performance model uncertainty. These results illustrate the significance of considering the influence of numerical error and performance uncertainty when performing a weather routing study.

Keywords: Uncertainty analysis, Numerical error, Weather routing
\end{abstract}

\section{Introduction}

Marine weather route modelling aims to identify a route where variables such as time, fuel usage or risk, amongst others are optimised given the performance model and the weather that is expected on a given route. Within the context of sailing craft weather routing, the most common optimised variable is time.

Route modelling can be used within the design process for new designs, such as 
in sailing craft hull design [1], or operationally, in order to provide routes for existing craft. When applied operationally, weather routing is essential for improving the safety of mariners at sea through identifying routes which minimise fuel costs, risk or time.

Quantifying the significance of uncertainty in the weather routing process allows the credibility of the predictions to be quantified, managing the expectations of the operators with regards to the accuracy of the supplied route. Key sources of uncertainty are the weather data, the accuracy of the performance model and the numerical error in the solution of the optimisation algorithm. However, research has not investigated the quantification of the numerical error of the routing algorithm or the impact of uncertainty in the performance model. The increasing availability of high performance computing and associated improvement of programming languages allows previously computationally intractable problems to be simulated.

This paper introduces a methodology for quantifying the significance of numerical error and performance uncertainty in a marine weather routing study. Initially the numerical error of the optimisation algorithm will be quantified through varying the discretization of the environment. The influence of uncertainty of the performance is estimated through varying the performance linearly about the original performance model.

A marine weather routing problem with a high level of uncertainty is the modelling of ethnographic voyaging canoes completing colonisation voyages across Polynesia. This problem involves predicting the voyaging time of a sailing craft over a given route for a range of weather conditions, a typical design problem. It is possible to model the performance of any marine craft as a function of wind and wave conditions, allowed this method to be applied to any marine craft.

\subsection{Literature review}

This review will discuss the literature on sailing craft performance prediction and weather routing algorithms. The weather routing process predicts the optimum route for a sailing craft to take between two points. The method considers the performance model of the sailing craft, the optimisation algorithm and the environmental data used to identify the optimum path. 
The performance of a sailing craft is determined by the balance of the driving force generated by the wind passing over the sail against the resistance of the hull and appendages. The wind passing over the sail also generates a heeling moment which is balanced against the righting moment of the hull. The interaction of these key force balances and the additional moments determine the speed and heel angle of the sailing yacht [2].

Static and dynamic velocity prediction programs (VPPs) are used to model the performance of a sailing craft. Static VPPs predict the conditions at which the forces and moments acting on a sailing craft are balanced [2]. Dynamic VPPs evaluate the forces acting on the sailing craft over a series of time steps, within the context of a short race [3]. Static VPPs require less information than dynamic VPPs on the design of a sailing craft to provide performance predictions but are considered to be less accurate as a result.

The first research into solving the sailing craft route optimisation problem used a recursive dynamic programming formulation which divided the domain into nodes over which the minimum time path was calculated [4]. Different wind models [3, 5] or race strategy and opponent models [6, 7] have been used to improve the accuracy of sailing routing models.

The influence of different methods of modelling the ability for a sailing craft to sail upwind has been explored [8] along with modelling the time taken to complete course changes [9]. Sailing craft race modelling has typically minimised either the time taken to complete a course [10], risk of losing to an opponent [11, 6] or reliability [12]. However, the majority of sailing craft routing research only applies to a minimum time route sailed over a small spatial and temporal domain. Research has considered other factors such as risk [13], energy efficiency [14] and fuel and comfort [15].

The long course modelling problem can be characterised through its consideration of larger spatial and temporal domains over which the shortest path is solved. Typical drawbacks of short course routing methods involve their requirement for a predictable wind field and the requirement for the entire course to be modelled as being flat with respect to the curvature of the earth. The haversine formula becomes accurate at distances longer than $1 \mathrm{~nm}$ [4], this provides an indication at the crossover between short 
and long course routing methods.

The marine weather routing literature has developed a range of different shortest path algorithms. The different approaches were classified into calculus of variations, grid based approaches and evolutionary optimisation [16]. A time dependent approach based on the calculus of variations solved the shortest path problem through identifying the shortest path to take through considering a series of fronts reached within incremental time steps [17]. Forward dynamic programming approaches involve recursively solving the shortest path over a grid of locations generated along the great circle line between the start and finish locations [4]. This approach can be two dimensional or three dimensional considering how the variables such as time and fuel cost are optimised [18]. A claimed improvement is to use a floating grid system which updates potential locations every time step [19]. Using a 3D forward dynamic programing method it was possible to optimise energy efficiency through varying the engine power and ship heading [20]. These grids can be generated based off local wind conditions [7], although this approach would be challenging for larger spatio-temporal domains as found in long course routing.

Another route modelling approach has involved generating candidate routes using biased Rapidly exploring Random Trees which solve for the minimum energy path using the $\mathrm{A}^{*}$ algorithm [21]. A multi objective genetic algorithm has been applied to solve for the optimum path considering multiple safety and fuel constraints [22]. A real coded genetic algorithm allowed the consideration of ship rolling within the route optimisation process, thereby improving safety [23]. An improvement came with the implementation of a fuzzy logic model to model the performance of a sail driven vessel [24].

A method of iteratively aggregating the minimum time path over multiple weather scenarios has been introduced [4]. The approach of using ensemble weather scenarios has been shown to be more accurate than single scenarios with application to marine weather routing [25]. Multiple algorithms and objective functions have been implemented and applied to the marine weather routing problem. To the authors knowledge there has been no study of how uncertainties within the solution algorithm and objective function influence the confidence that may be had in the final result. 


\subsection{Uncertainty analysis} optimal path based on the discretization of the environment. Typical discretization error calculation methods require grids of significantly different sizes to be solved for a given set of initial conditions [31]. Through examining the rate of convergence of solutions from different grids it is possible to extrapolate the solution for an infinite tem are given a lack of knowledge on how certain parts of the system operate. The choice of how to categorize and thus simulate uncertainty is significant in the scientific modelling process [26]. In the marine environment it is possible to classify uncertainty as being aleatory uncertainty or epistemic uncertainty [27]. Aleatory uncertainty is the inherent randomness in a particular parameter, it is not possible to reduce this. Epistemic uncertainty is knowledge based, it is possible to reduce this quantity through collecting more information on the process in question. Epistemic uncertainty can be broken down into data uncertainty, statistical uncertainty, model uncertainty and climate uncertainty. Data uncertainty is associated with the error associated with collecting data from experiment or model. Statistical uncertainty is a consequence of not obtaining enough data to model a given phenomenon. Climate uncertainty addresses the ability for the climate variables over a given spatial-temporal domain to be representative given the nature of the weather and climate change [28].

The weather has been described as a chaotic process [29]. The chaotic nature of the weather limits the ability to utilise numerical models to predict into the future or to model what occured in the past. A chaotic system can be thought of as one where the present state determines the future state, but the approximate present state doesn't determine the approximate future. The use of ensemble weather scenarios generated using different intial conditions attempts to mitigate the associated uncertainty with weather forecasts and reanalysis data [30]. Given that the weather is a chaotic process, it is likely that any solution process for the minimum time path sequential decision making process may identify one or more stable solutions. Currently the simplest method of simulating weather uncertainty is through using past weather data.

The error in a scientific model is the accuracy at which it estimates a real system. The key source of error is the ability for the optimisation algorithm to identify the 
discretization.

\section{Method}

Figure 1 shows the method used in this research. The initial conditions such as the route and environmental conditions are specified. A suitable routing algorithm is identified and implemented. The numerical error is then estimated and the influence of performance uncertainty is modelled. The method is concluded with an analysis of the impact of performance uncertainty and numerical error on the interpretation of the final set of voyaging time results.

\subsection{Routing model}

This paper uses the Dynamic Programming (DP) algorithm as applied to long distance sailing craft in [4], the simplicity of this algorithm means that the source of uncertainty lies in the discretization of the domain over which the minimum time path is solved.

We consider a large domain on the Earths surface over which the modelled sailing craft could hypothetically sail. This domain is discretized into an equal number of locations both in parallel and perpendicular to the Great Circle drawn between the start and finish locations. The distance between each location is controlled through defining the maximum distance between nodes, $d_{n}$. $d_{n}$ can be controlled seperately as the grid height or grid width, as seen in Figure 2 for this research it is set as being equal. This generates a grid of nodes with equal numbers of ranks as well as nodes within each rank.

For the position at any given node $i$ the travel time between nodes $i$ and a node on the next rank $j$ along the arc $(i, j)$ starting at time $t$ is $c_{\text {arc }}(i, j, t)$. The cost function, $c_{\text {arc }}(i, j, t)$, provides an estimate of the time taken for a sailing craft to sail between two points given the environmental conditions at time $t$. An intial speed estimate is taken from interpolating the results of a performance prediction analysis for the specific wind condition. This speed is then modified in order to account for the wave conditions being experienced. A final optimisation takes place to identify the optimum heading to sail 


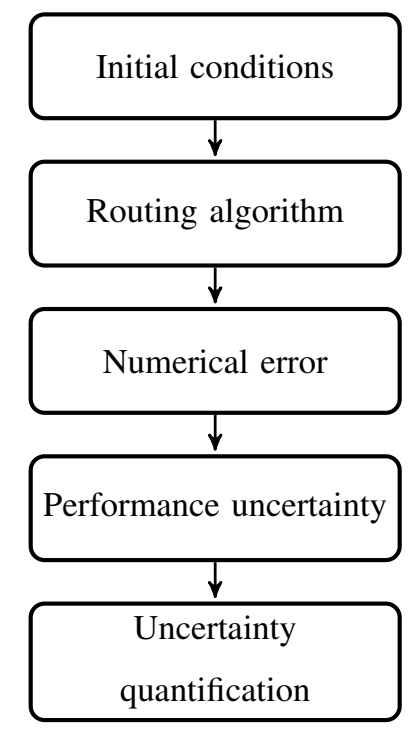

Figure 1: Method used to quantify uncertainty in marine weather routing.

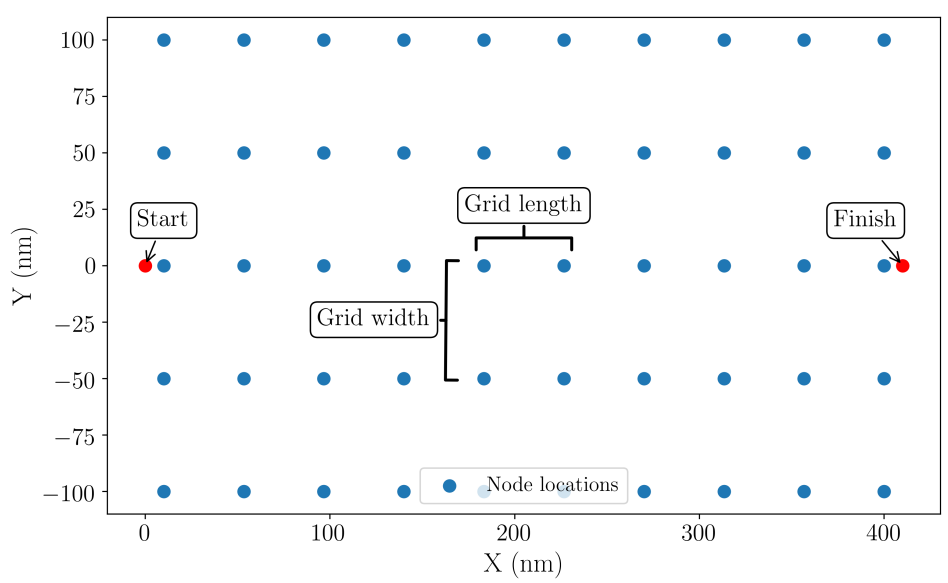

Figure 2: Discretized domain along great circle line between voyage start and finish.

between the two points given the current the craft is exposed to. If it is not possible to achieve a positive speed towards location $i$ from location $j$ then the speed will be set to 0 returning an infinite travel time for that particular arc. It is possible to penalise areas of the domain in this manner and identify combinations of initial conditions which are 
unable to return

The minimum time path is identified using a forward looking recursive algorithm, described in Equation 1 $f^{*}(i, t)$ is the time taken for the optimal sequence of decisions from the node-time pair $(i, t)$ to the finish node and $j^{*}(i, t)$ is the successor of $i$ on the optimal path when in state $t$. Through solving $j^{*}(i, t)$ within $f^{*}(i, t)$ it's possible to solve for the minimum time path. $\Gamma_{i}$ is the set of all nodes on the graph. The algorithm iterates from the start node to the finish node and updates each node in between with the earliest time that it can be reached.

$$
\begin{array}{r}
f^{*}(i, t)= \begin{cases}0, & i=n_{\text {finish }} \\
\min _{j \in \Gamma_{i}}\left[c_{\text {arc }}(i, j, t)+f^{*}\left(j, t+c_{\text {arc }}(i, j, t)\right)\right], & \text { otherwise }\end{cases} \\
j^{*}(i, j)=\arg \min _{j \in \Gamma_{i}}\left[c_{\text {arc }}(i, j, t)+f^{*}\left(j, t+c_{\text {arc }}(i, j, t)\right)\right], i \neq n_{\text {finish }}
\end{array}
$$

The weather data used is from the ERA20 climate model has a spatial resolution of $125 \mathrm{~km}$ and a temporal resolution of 3 hours [32]. The weather data is linearly in- 
tepolated to the discretization of the nodes in the environment. If the distance between nodes is much larger than the original spatial discretization of the weather data then the change in the weather conditions will not be fully modelled. The voyaging time is used to look up the closest weather data time step.

The accuracy of the performance model is limited to certain scales, consequently it may not make physical sense to apply such a model below a certain length of time or distance. Despite this, a quantification of the numerical solution algorithm is still required in order to identify whether the minimum time solution is stable. One index used to quantify numerical uncertainty in CFD is the grid convergence index (GCI) [31,33] which has only recently been applied to the sailing craft routing problem [12].

The method of numerically quantifying error using the Grid Convergence Index (GCI) is fully described in [33]. It involves the solution of the algorithm over multiple discretizations of the environment which exponentially increase in detail. The grid height, $h$, is the measurement unit of grid size and is calculated using Equation $2 \Delta A_{i}$ is the size of the $i$ th cell and $N$ is the total number of the cells used for computation. The solution trend from the three distinct grid sizes is extrapolated towards $h \rightarrow 0$ where the extrapolated solution is used to estimate the associated discretization error.

$$
h=\left[\frac{1}{N} \sum_{i=1}^{N}\left(\Delta A_{i}\right)\right]
$$

We wish to quantify whether uncertainty surrounding the performance modelling of a sailing craft is significant within the context of sailing craft route modelling. To begin the process the performance model is varied linearly about its original performance value. Each generated performance model is known as $P_{\text {unc }}$, where unc is the percentage that the original performance is varied by.

Algorithm 1 describes the uncertainty simulation routine for a specific route at a specific time. It shows how the minimum time, $V_{t, d_{n}, \text { unc }}$, for a specific combination of start time $t$, height $d_{n}$, and performance uncertainty level $u n c$ is simulated for a given route. A range of grid heights, $h_{n}$, are selected based on studying how the estimates from the routing algorithm converge as the height is reduced. The lower limit of $d_{n}$ is 
The number of start times and uncertain performances to be simulated is dependent on the computational resources available. The code for this method was implemented in the programming language Julia [34].

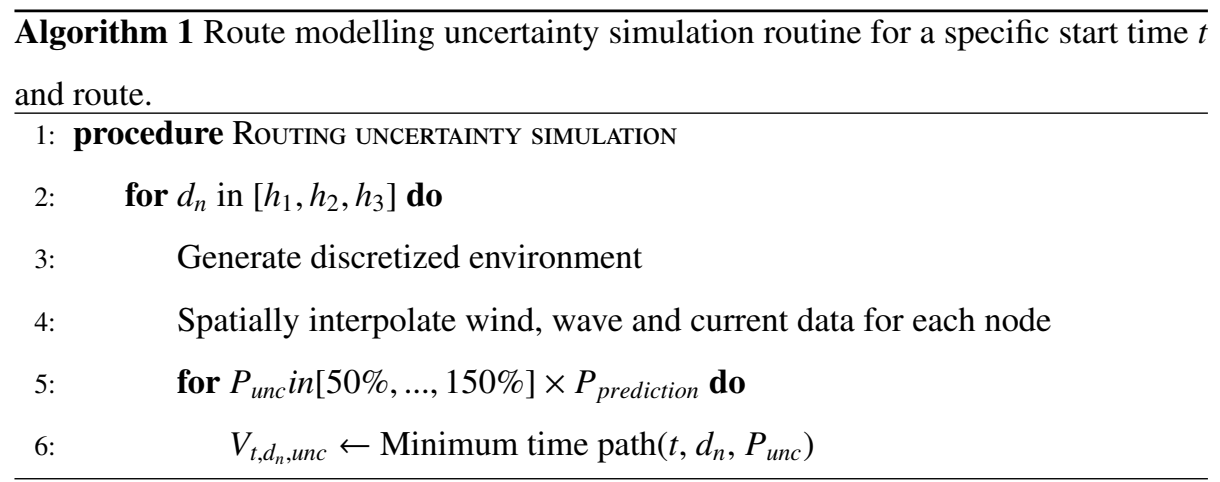

\section{Application}

The uncertainty route modelling analysis procedure is applied to quantifying the performance of Polynesian voyaging canoes, an application with previously irreducible levels of uncertainty. Modelling the voyaging time for ethographic voyaging craft to complete specific routes will assist understanding how it was possible for Polynesia to be colonised, one of Pacific archaeology's greatest unanswered questions [35]. Of interest is the influence of the ENSO oscillation, a key weather phenomenon in the Pacific, on the voyaging time [36]. One voyaging route of interest is between Upolu and Moorea. Through simulating the influence of performance uncertainty on the voyaging time for a colonisation voyage it will be possible to quantify the influence of seafaring technology on the rate of the colonisation of Polynesia. At the heart of this problem is ${ }_{230}$ the solution of a marine weather routing algorithm with a prior requirement to simulate uncertainty.

The minimum time path between Tongatapu and Atiu was estimated for a range of different grid sizes, performances and start times. The results from this series of simulations can be used to estimate the difficulty of making the voyage between these two locations using prehistoric seafaring technology. Twenty different performances were 

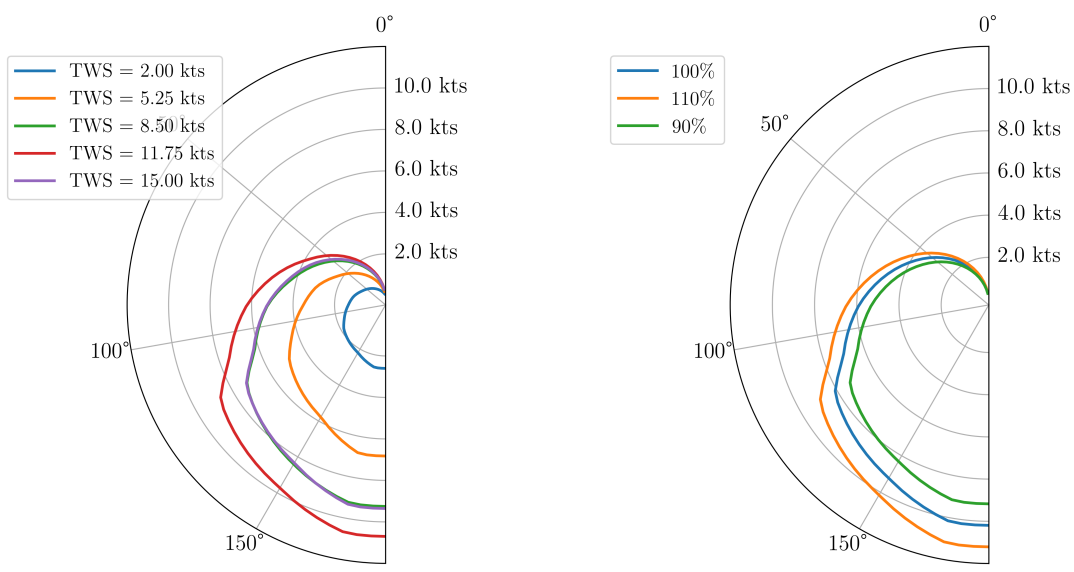

(a) $V_{s}$ as a function of true wind speed and direction for the (b) $V_{s}$ varied $10 \%$ about the original performance for a wind sailing craft 37 . speed of $10 \mathrm{kts}$.

Figure 3: Performance of marine vessel used in study. The polar plots show the speed achieved by the vessel for a specific true wind angle at different performance levels or true wind speeds.

generated linearly varying from $-50 \%$ to $+50 \%$ of the original performance model. Voyages were started every 72 hours from the 1st January to the 31st December. 1985 is used to provide weather data for a medium ENSO, an important condition in the context of the study.

To quantify the efficacy of later voyaging canoe designs, performance data for an outrigger canoe was used. This performance was estimated based off CFD simulations for the hull configuration and aerodynamic experiments for the sail. The full details for the performance prediction may be found in [37]. It should be noted that it is possible to substitute in any performance model within this framework, allowing application to all marine vehicles.

The cost function for this craft interpolates the performance from the polar performance diagram, seen in Figure $3 \mathrm{a}$. An example of how the performance is varied for a specific wind condition is shown in Figure $3 b$. The wind and wave reanalysis data was downloaded for the year 1982 from the ECMWF ERA20 model [32]. The current data used was sourced from [38]. 


\subsection{Numerical uncertainty}

\subsubsection{Illustration of simulation convergence}

The numerical error in the routing algorithm is a function of the discretization of the domain, parameterised by the grid width, $d_{n}$. Figure 4 illustrates the predicted routes between Tongatapu and Atiu where $d_{n}$ was reduced in stages between 40.0 to 5 $\mathrm{nm}$. It can be seen that as the fidelity of the simulation increases, the voyaging time, $V_{t}$, reduces.

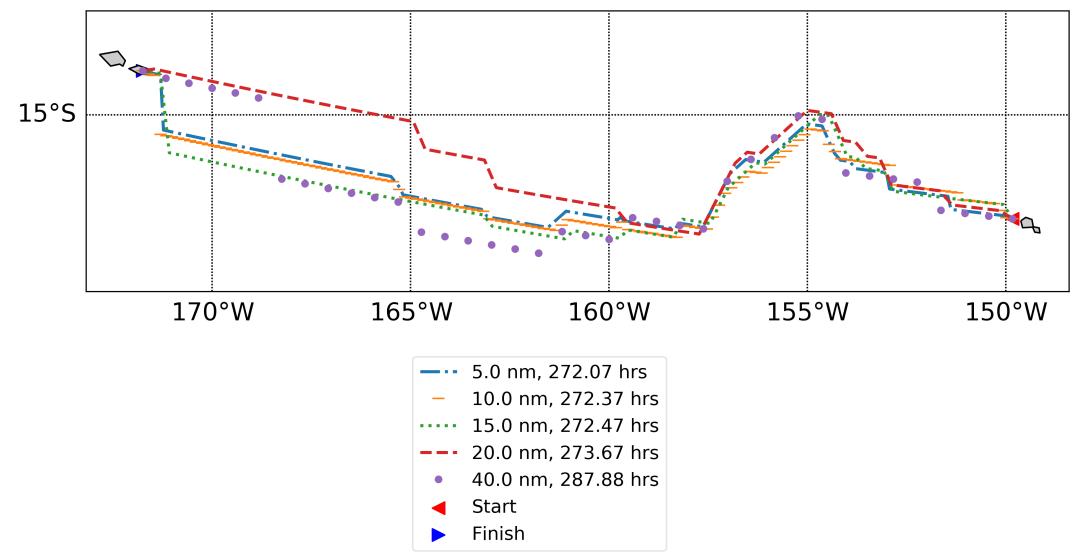

Figure 4: Routes between Upolu and Moorea starting at 00:00 GMT on the 1st January 1985 solved over several different grid widths.

A convergence plot of $V_{t}$ as a function of $d_{n}$ is shown in Figure 5 For the specific initial conditions the relationship is that as $d_{n}$ reduces, so too does $V_{t}$. It can be seen

that there is a significant change in relationship between the results for $d_{n}=40,20$ and $d_{n}=15,10,5 \mathrm{~nm}$ and $V_{t}$. This could be due to the grid discretization becoming significantly finer than the resolution of the original wind and wave data.

The extrapolated $V_{t}, \mathrm{GCI}$ and order of convergence for three combinations of grid height is included in Table 1 The extrapolated $V_{t}$ value is where the predicted $V_{t}$ for the specified heights is extrapolated to $d_{n}=0$. The solution for $d_{n}=10.0,15.0,20.0$ has a low GCI indicating the results for each of these grid heights appear to converge. However, we see a slight increase in $V_{t}$ and GCI for $d_{n}=5.0,10.0,15.0 \mathrm{~nm}$. This data suggests a positive correlation between between $V_{t}$ and GCI, but also that there is a 


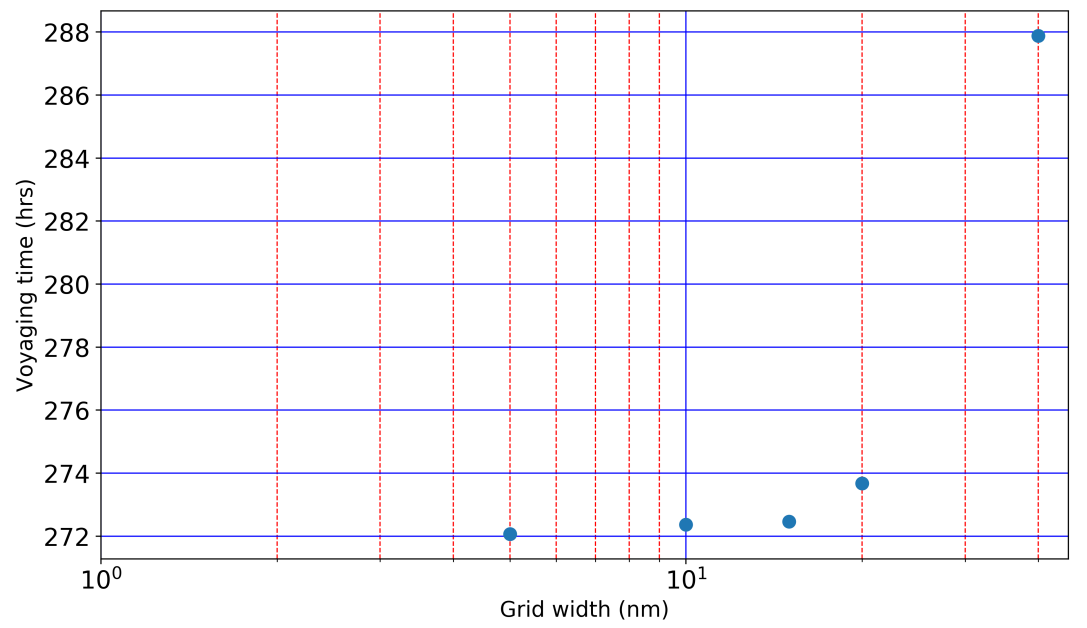

Figure 5: Voyaging time, $V_{t}$, as a function of grid width for voyages between Upolu and Moorea starting at 00:00 GMT on the 1st January 1985.

relationship between $d_{n}$ and GCI which must be considered to qualify the results.

The resolution of the weather data could contribute towards inaccuracy at lower values of $d_{n}$. The resolution of the weather data is at $67.49 \mathrm{~nm}$, and the results may also suggest that as $d_{n}$ decreases significantly below the resolution of the weather data the solutions may become dependent on the interpolation techniques used to retrieve the weather data.

There is a large computational cost associated with finer simulations which prohibits the number of simulations required to explore uncertainty in voyaging time. These results show that the GCI calculation method for estimating numerical error must be applied in a manner considering the physical implication of the parameters but also the computational run time of the simulations.

The selection of $d_{n}$ is a compromise between simulation accuracy and computational run time. Simulation accuracy is determined by the smallest and the largest scales that the cost function can be applied to. The smallest scale is determined by the haversine formula which has significant levels of error for distances below $1 \mathrm{~nm}$. The largest scale is determined by the rate at which spatio-temporal environmental data becomes available. Parallel computing provides significantly more resources than 


\begin{tabular}{llll}
\hline$d_{n}$ & $V_{t}$ (hrs) & GCI & OOC \\
\hline $5.0,10.0,15.0$ & 274.00 & 0.0014577 & 2.25 \\
$10.0,15.0,20.0$ & 272.49 & 0.0000955 & 4.34 \\
$15.0,20.0,40.0$ & 272.64 & 0.0012429 & 2.59 \\
\hline \hline
\end{tabular}

Table 1: Calculation of extrapolated $V_{t}$, GCI and order of convergence.

available previously allowing an increase in the number of different initial conditions that are required to be simulated. However, there are still large costs associated with the finer simulations at $d_{n}=2.5,5$.

Figure 5 illustrates that as $d_{n}$ is reduced significantly below the spatial distribution of weather data there is a step change in the solution. The $d_{n}$ also influences the rate at which new weather data is retrieved and processed. If the journey time for a particular arc between two nodes lasts longer than the time between weather conditions being updated then the solution is being solved over incomplete information. The desire for accuracy must be balanced against computational limitations. There is a cubic relation between the computational run time and fidelity of the simulation. From this set of initial simulations it may be proposed that the $d_{n}=10.0,15.0,20.0 \mathrm{~nm}$ provide a collection of heights which balance the requirement for accuracy against computational run time.

\subsubsection{Numerical error of voyaging simulations}

The numerical error from the application of the routing algorithm must be calculated to qualify the solution. Each set of initial conditions require a new estimate of numerical error. The average numerical error across a range of simulations will give insight, and confidence, into how well the routing algorithm is able to solve across a range of initial conditions.

The order of convergence, GCI value and extrapolated voyaging time were calculated for the times generated as a function of the different grid sizes of 10, 15 and 20 $\mathrm{nm}$. The order of convergence measures the rate at which the difference between the magitude of each result changes as a function of the change of the grid width. $95.66 \%$ 
of the remaining results had an order of convergence greater than 1.0, a necessary rerequiring more analysis.

The relationship between $V_{t}$ and $G C I$ is shown in Figure 6 From the fitting of the line to the data it can be seen that there is no relationship between $V_{t}$ and $G C I$. This is because the same number of calculations are being performed over each grid sign problem, albeit one with large levels of uncertainty. Voyages between Tongatapu and Atiu were started every 6 hours from the 1st January 1985 to the 31st December 1985. The performance model was varied for 21 steps between $50 \%$ and $150 \%$ of the original performance. Simulations were solved over a grid size of $d_{n}=10 \mathrm{~nm}$. Signif335 changes in performance, as seen in Figure 7

The mean voyaging time appears to be between 230 - 295 hours for the unaltered performance with the variation being solely due to the range of environmental condi- 


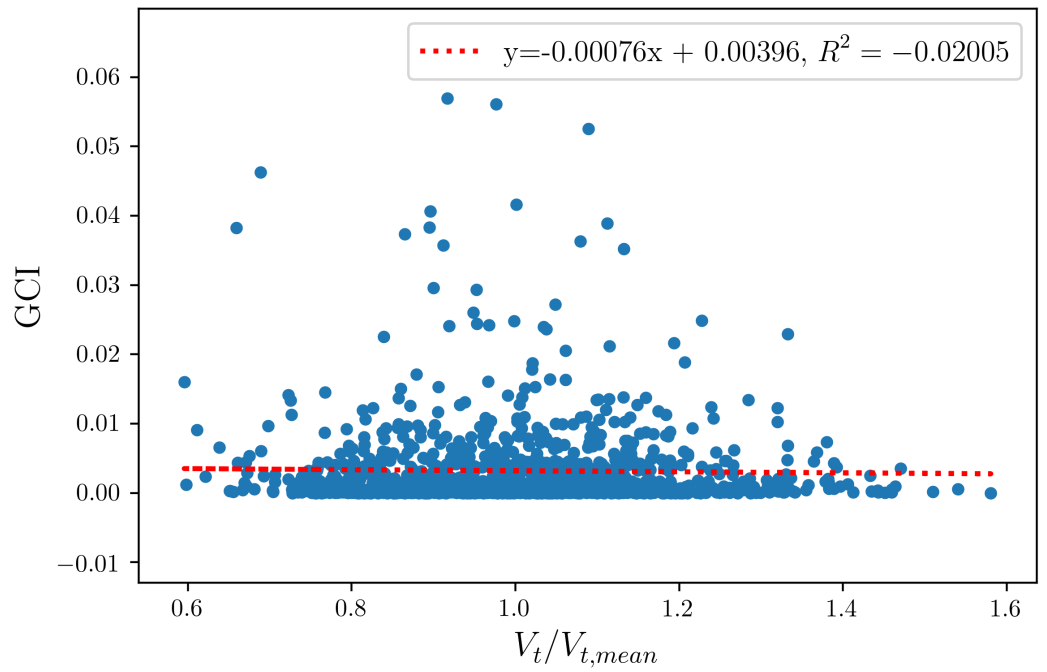

Figure 6: Relationship between GCI index and voyaging time.

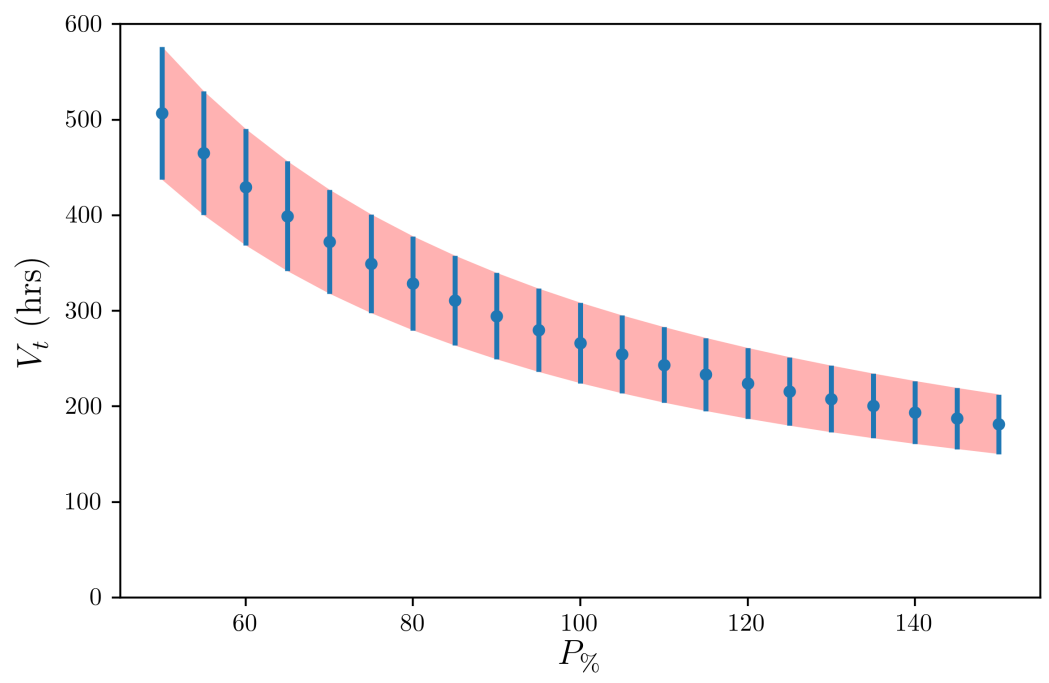

Figure 7: Voyaging time as a function of the different variations of performance. The standard deviation is illustrated using error bars.

tions. This illustrates that even if there was a high level of confidence associated with

the accuracy of the performance model the weather conditions contribute signficantly 
to the variation of voyaging time.

A large variation in voyaging time can be seen in the results. The minimum voyaging time is 98.42 hours, with a maximum of 682.77 hours. Increasing uncertainty in the accuracy of the performance model contributes to the variation in voyaging time. 345 To reduce this uncertainty the accuracy of the performance model must be quantified before performing a routing study so it is possible bound the uncertainty associated with the minimum voyaging time.

Of interest is the variance of the voyaging time as a function of the performance variation. Understanding how as the varying of performance influences the voyaging time indicates the degree of confidence that should be held in a given voyaging result, given the confidence in the performance mode.

Figure 8 shows the relationship between performance variation and the voyaging time non-dimensionalised with respect to the original performance voyaging time for each start date. This illustrates how variations in performance from the original performance significantly alter the expected voyaging time. It can be seen that as the performance varies from the initial performance the standard deviation of the voyaging time results increases. As time is a reciprocal of speed it can be seen that the magnitude of the reduction of voyaging time in response to performance improvement is smaller than the magnitude of the increase of voyaging time in response to performance reduction.

There is also a difference between the change in the standard deviation of $V_{t}$ for equivalent magnitude variations about the original performance. As the performance decreases we see more significant reductions in speed and much larger increases in standard deviation. As the performance increases the magnitude between successive improvements decreases along with a reduction in standard deviation. It can be seen that reductions in performance have more significant negative impacts on voyaging time than equivalent improvements. This non-linear response is due to the slower craft spending more time at sea and consequently being exposed to more variation in the weather.

Figure 9 shows how the standard deviation of the voyaging time varies as a function of the performance variation. The average numerical is overlaid to provide an indication of how significant it may be when using the results of this study. Figure 9 indicates 


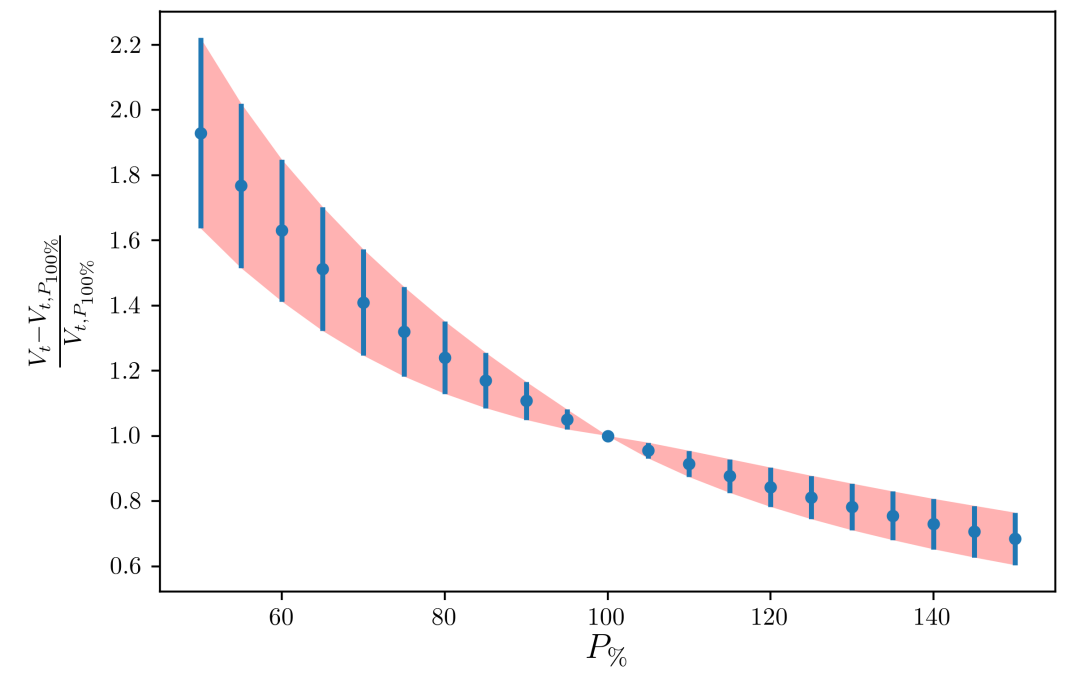

Figure 8: Relationship between the voyaging time and change in performance. The standard deviation is plotted as the error bars.

that the contribution of performance uncertainty is much larger than the numerical error of the algorithm. A variation in performance of $\pm 2.5 \%$ causes a standard deviation of $0.8-1.1 \%$, equivalent to $2.13-2.93 \mathrm{hrs}$ for the mean voyaging time of $266.40 \mathrm{hrs}$. As the variation from the original performance increases to $\pm 5 \%$ we see that the standard deviation increases rapidly to $2.41-3.08 \% V_{t}$, or, $6.42-8.21$ hours.

These results illustrate the influence of performance uncertainty on $V_{t}$. It is likely that the uncertainty associated with forecast data is still the dominant source of uncertainty, as has been specified earlier in this paper.

The weather conditions are updated every 3 hours. The magnitude of the numerical error and influence of low levels of performance uncertainty indicate that it is possible for multiple changes in the weather to not be modelled. It would be difficult to quantify the impact of this error on the ability for the weather routing model to approximate the real situation. 


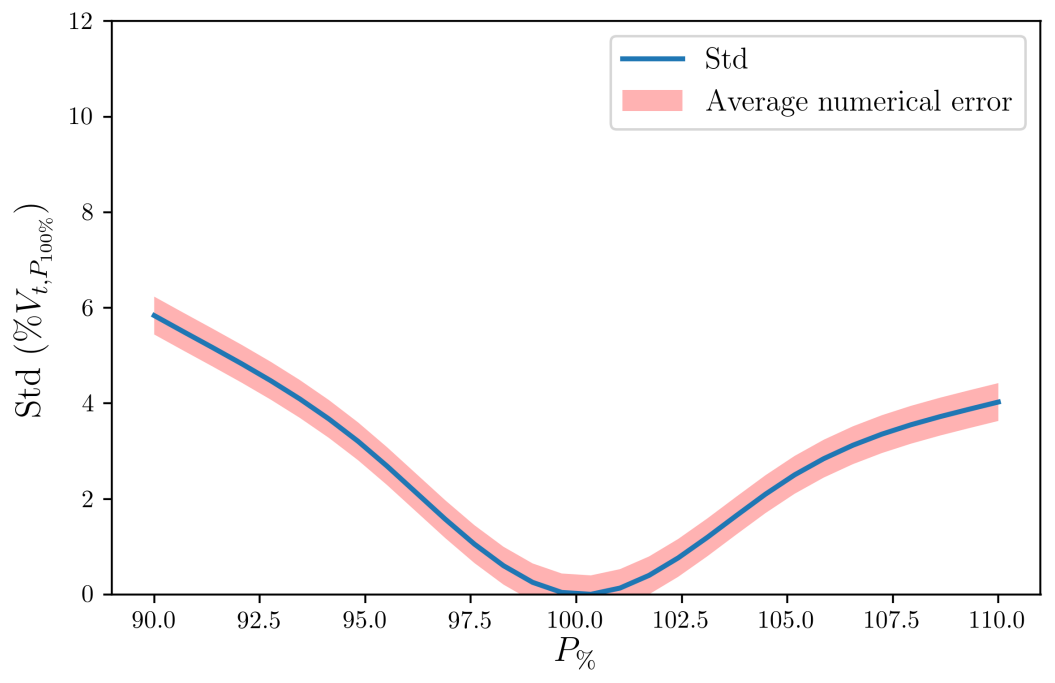

Figure 9: Relationship between performance variation and the standard deviation of $V_{t, P_{100 \%}}$.

385

\section{Summary and conclusions}

This paper has presented a method for evaluating the impact of numerical error and performance variation in the voyaging time for a marine weather routing problem. This method was applied to a typical design problem; the quantification of the time taken for a sailing craft to complete a specific voyage given uncertainty in its performance. The key results of this study can be summarised as follows;

1. Uncertainty in performance and the numerical error reduce the accuracy of marine weather routing, the dominant source of uncertainty is likely to lie with meteorological uncertainty for applications relying on forecast data.

2. The numerical error must be calculated for each set of initial conditions. For this problem, $95.3 \%$ of all simulations converged with an average of $0.320 \%$ error. For the slowest performance the mean voyaging time is 506.92 with a numerical error of \pm 1.62 . For the fastest performance the mean voyaging time is $181.38 \pm 0.58$ hrs.

3. Slower craft spend more time at sea they are exposed to more variance in the 400 weather conditions, likely contributing towards the non-linear response of voy- 
aging time to performance variation. This means that the uncertainty in the performance model must be quantified to provide credibility to voyaging simulations.

4. The influence of uncertainty in the performance model rapidly becomes more influential than the routing algorithm numerical error. The relationship between uncertainty and the standard deviation of voyaging time increases sharply with variations of $2.5 \%$ in performance being associated with standard deviations of $\pm 0.8-1.1 \%$ about the mean voyaging time.

5. The weather data used updates every 3 hours. The combination of numerical error and uncertainty in performance model may mean that the approximation of the minimum time path is being calculated based off incomplete sets of weather data, or solved using more weather data than would be encountered in practice.

This method of quantifying the numerical error of the solution algorithm and performance uncertainty could be applied to other cases involving marine vessels such as cargo ships. This would allow an understanding of the maximum level of accuracy that could be achieved within commerical practice. Another investigation could be into the uncertainty levels associated with the recorded reanalysis weather data used and how this might influence the result.

Through applying an uncertainty analysis method to the marine weather routing problem we have shown that the influence of performance uncertainty is much larger than any uncertainty associated with the optimisation algorithm used. To provide more accurate routing the uncertainty associated with the performance model used must be reduced.

\section{Acknowledgements}

Dr Gabriel Weymouth, Professor Dominic Hudson and Dr Blair Thornton for discussion of methodology and results. Carlos Losada de la Lastra for extensive comments on the text. A special thanks to the contributions made by the reviewers who provided significant improvements to the original text. 


\section{Funding sources}

${ }_{430}$ This work was funded by the Southampton Marine and Maritime Institute and the University of Southampton.

\section{References}

[1] J. Oliver, Hull design and evaluation, in: A. Claughton, J. Wellicome, R. Shenoi (Eds.), Sailing Yacht Design: Practice, 2nd ed., University of Southampton, Southampton, 2006, pp. 101-124.

[2] A. B. Philpott, R. M. Sullivan, P. S. Jackson, Theory and Methodology Yacht velocity prediction using mathematical programming, European Journal of Operational Research 67 (1993) 13-24.

[3] A. B. Philpott, S. G. Henderson, D. Teirney, A Simulation Model for Predicting Yacht Match Race Outcomes, Operations Research 52 (2004) 1-16.

[4] T. Allsopp, Stochastic Weather Routing for Sailing Yachts (1998) 110.

[5] R. C. Dalang, F. Dumas, S. Sardy, S. Morgenthaler, J. Vila, Stochastic optimization of sailing trajectories in an upwind regatta, Journal of the Operational Research Society 66 (2015) 807-821.

445

[6] T. B. Spenkuch, A Bayesian Belief Network Approach for Modelling Tactical Decision-Making in a Multiple Yacht Race Simulator By, Ph.D. thesis, University of Southampton, 2014.

[7] F. Tagliaferri, I. M. Viola, A real-time strategy-decision program for sailing yacht races, Ocean Engineering 134 (2017) 129-139.

[8] R. Stelzer, T. Pröll, Autonomous sailboat navigation for short course racing, Robotics and Autonomous Systems 56 (2008) 604-614.

[9] S. P. Ladany, O. Levi, Search for optimal sailing policy, European Journal of Operational Research 260 (2017) 222-231. 
[10] R. Ferretti, A. Festa, A Hybrid control approach to the route planning problem for sailing boats, Http://Arxiv.Org/Abs/1707.08103 (2018) 1-27.

[11] F. Tagliaferri, A. B. Philpott, I. M. Viola, R. G. Flay, On risk attitude and optimal yacht racing tactics, Ocean Engineering 90 (2014) 149-154.

[12] T. Dickson, J. Blake, D. Sear, Reliability informed routing for Autonomous Sailing Craft, in: S. Schillai, N. Townsend (Eds.), International Robotic Sailing Conference, Southampton, 2018.

[13] A. Decò, D. M. Frangopol, Risk-informed optimal routing of ships considering different damage scenarios and operational conditions, Reliability Engineering and System Safety 119 (2013) 126-140.

[14] R. Zaccone, Energy Efficient Ship Voyage Planning By 3D Dynamic, Journal of Ocean Technology 12 (2017).

[15] R. Zaccone, E. Ottaviani, M. Figari, M. Altosole, Ship voyage optimization for safe and energy-efficient navigation: A dynamic programming approach, Ocean Engineering 153 (2018) 215-224.

[16] L. Walther, A. Rizvanolli, M. Wendebourg, C. Jahn, Modeling and Optimization Algorithms in Ship Weather Routing, International Journal of e-Navigation and Maritime Economy 4 (2016) 31-45.

[17] S. Bijlsma, On Minimal-Time Ship Routing., Phd, Delft, 1975.

[18] H. Hagiwara, J. A. Spaans, Practical Weather Routing of Sail-assisted Motor Vessels, Journal of Navigation 40 (1987) 96-119.

[19] Y.-H. Lin, M.-C. Fang, R. W. Yeung, The optimization of ship weather-routing algorithm based on the composite influence of multi-dynamic elements (II): Optimized routings, Applied Ocean Research 43 (2013) 184-194.

[20] W. Shao, P. Zhou, S. K. Thong, Development of a novel forward dynamic programming method for weather routing, Journal of Marine Science and Technology (Japan) 17 (2012) 239-251. 
[21] D. Rao, S. B. Williams, Large-scale path planning for Underwater Gliders in ocean currents, in: Australasian Conference on Robotics and Automation, January 2009, Sydney, 2009.

[22] J. Hinnenthal, Robust Pareto-Optimum Routing of Ships utilizing Deterministic and Ensemple Weather Forecast (2008).

[23] A. Maki, Y. Akimoto, Y. Nagata, S. Kobayashi, E. Kobayashi, S. Shiotani, T. Ohsawa, N. Umeda, A new weather-routing system that accounts for ship stability based on a real-coded genetic algorithm, Journal of Marine Science and Technology 16 (2011) 311-322.

[24] S. Marie, E. Courteille, Sail-assisted motor vessels weather routing using a fuzzy logic model, Journal of Marine Science and Technology (Japan) 19 (2014) 265279.

[25] L. Skoglund, J. Kuttenkeuler, A. Rosén, E. Ovegård, A comparative study of deterministic and ensemble weather forecasts for weather routing, Journal of Marine Science and Technology (Japan) 20 (2015) 429-441.

[26] A. Der Kiureghian, O. Ditlevsen, Aleatoric or Epistemic? Does it matter?, Special Workshop on Risk Acceptance and Risk Communication (2007) 13.

[27] E. M. Bitner-Gregersen, S. K. Bhattacharya, I. K. Chatjigeorgiou, I. Eames, K. Ellermann, K. Ewans, G. Hermanski, M. C. Johnson, N. Ma, C. Maisondieu, A. Nilva, I. Rychlik, T. Waseda, Recent developments of ocean environmental description with focus on uncertainties, Ocean Engineering 86 (2014) 26-46.

[28] E. M. Bitner-Gregersen, $\varnothing$. Hagen, Uncertainties in data for the offshore environment, Structural Safety 7 (1990) 11-34.

[29] E. N. Lorenz, Deterministic Nonperiodic Flow, Journal of the Atmospheric Sciences 20 (1963) 130-141.

[30] J. Slingo, T. Palmer, Uncertainty in weather and climate prediction, Philosophical Transactions of the Royal Society A: Mathematical, Physical and Engineering Sciences 369 (2011) 4751-4767. 
[31] P. J. Roache, Quantification of Uncertainty in Computational Fluid Dynamics, Annual Review of Fluid Mechanics 29 (1997) 123-160.

[32] P. Poli, H. Hersbach, D. P. Dee, P. Berrisford, A. J. Simmons, F. Vitart, P. Laloyaux, D. G. Tan, C. Peubey, J. N. Thépaut, Y. Trémolet, E. V. Hólm, M. Bonavita, L. Isaksen, M. Fisher, ERA-20C: An atmospheric reanalysis of the twentieth century, Journal of Climate 29 (2016) 4083-4097.

[33] I. B. Ghia, U. Celik, P. J. Roache, P. E. Raad, Christopher J. Freitas Hugh Coleman, P. E. Raad, Procedure for Estimation and Reporting of Uncertainty Due to Discretization in CFD Applications, Journal of Fluids Engineering 130 (2008) 078001.

[34] J. Bezanson, A. Edelman, S. Karpinski, V. B. Shah, Julia: A Fresh Approach to Numerical Computing, SIAM Review 59 (2017) 65-98.

[35] G. Irwin, R. Flay, Pacific Colonisation and Canoe Performance: Experiments in the Science of Sailing, The Journal of the Polynesian Society 124 (2015) 419444.

[36] Á. Montenegro, R. T. Callaghan, S. M. Fitzpatrick, Using seafaring simulations and shortest-hop trajectories to model the prehistoric colonization of Remote Oceania, Proceedings of the National Academy of Sciences 113 (2016) $12685-12690$.

[37] F. Boeck, K. Hochkirch, H. Hansen, S. Norris, R. G. J. Flay, Side force generation of slender hulls - influencing polynesian canoe performance, 4rd High Performance Yacht Design Conference (2012) 12-14.

[38] F. Bonjean, G. S. E. Lagerloef, Diagnostic Model and Analysis of the Surface Currents in the Tropical Pacific Ocean, Journal of Physical Oceanography 32 (2002) 2938-2954.

[39] ECMWF, (EFI) ROC skill, 10m wind speed, 2019. URL: https ://www .ecmwf . 535 int/\{\%\}OAen/forecasts/charts/catalogue/plwww\{_\}m\{_\}efi\{_\}roc? 
facets $=$ undef ined $\{\&\}$ time $=\{\%\} 0 A 2019021600\{\&\}$ parameter $=$

10mwindspeed. 